\title{
Nosocomial Outbreak of Extensively and Pan Drug-Resistant Acinetobacter baumannii in Tertiary Hospital in Central Part of Iran
}

\author{
Alireza Japoni-Nejad ${ }^{1,2}$, Masoomeh Sofian ${ }^{3,4}$, Alex van Belkum ${ }^{5,6}$,Ehsanollah Ghaznavi-Rad ${ }^{2,7^{*}}$ \\ ${ }_{2}^{1}$ Student Research Committee, Arak University of Medical Sciences, Arak, IR Iran \\ ${ }_{3}^{2}$ Department of Microbiology and Immunology, Faculty of Medicine, Arak University of Medical Sciences, Arak, IR Iran \\ 3 Department of Infectious Disease, Arak University of Medical Sciences, Arak, IR Iran \\ 4 Department of Infectious Disease, Arak University of Medical Sciences, Arak, IR Iran \\ Tuberculosis and Pediatric Infectious Research Center, Arak University of Medical Sciences, Arak, IR Iran
5 Department of Medical Microbiology and Infectious Diseases Erasmus MC, Rotterdam, The Netherlands \\ ${ }_{6}^{5}$ Department of Medical Microbiology and Infectious Diseases
${ }_{\text {Unit Microbiology, R\&D Microbiology, BioMerieux, France }}$ \\ ${ }_{7}^{6}$ Unit Microbiology, R\&D Microbiology, BioMerieux, France
Molecular and Medicine Research Center, Department of Microbiology, School of Medicine, Arak University of Medical Sciences, Arak, IR Iran \\ ${ }^{*}$ Corresponding author: Ehsanollah Ghaznavi-Rad, Department of Microbiology and Immunology, Faculty of Medicine, Arak University of Medical Sciences, Arak, IR Iran. Tel: +98- \\ 8614173526, E-mail: e.ghaznavirad@arakmu.ac.ir, ghaznaviehs@yahoo.com. \\ Received: December 26, 2012; Revised: February 20, 2013; Accepted: March 10, 2013
}

\begin{abstract}
Background: Acinetobacter baumannii has emerged as a cause of nosocomial infections in hospitalized patients, particularly in intensive care units. Carbapenems are a common choice for treating nosocomial infections caused by A. baumannii strains. Increasing antimicrobial resistance among Acinetobacter isolates has been documented and multidrug-resistant $A$. baumannii is recognized to be among the most difficult antimicrobial-resistant bacilli to control and treat.

Objectives: This study describes carbapenem resistance in A. baumannii isolates obtained during an outbreak from intensive care units of a peripheral hospital in central part of Iran.

Patients and Methods: Sixty-three non-repetitive A. baumannii isolates were collected over a six months period. Susceptibility of the isolated bacteria to a panel of 23 different antimicrobial agents was defined by using the standard disk diffusion method. Production of Metallo- $\beta$-lactamases (MBL) and AmpC $\beta$-lactamase were determined by using the E-test MBL strip and AmpC disk tests, respectively. Results: The present study indicates that carbapenems and new cephalosporin antibiotics were practically ineffective against the extensive drug resistance (XDR) strains. Colistin was observed to be more effective, although in seven cases resistance to colistin observed. AmpC $\beta$-lactamase and MBL could be an important contributory factor for imipenem resistance among the isolates in our hospital. The elevated prevalence of XDR and pan drug resistance(PDR) strains indicates that local antibiotic prescription policies should be revised and infection control should be improved.

Conclusions: The elevated prevalence of XDR and PDR strains indicates that local antibiotic prescription policies should be revised and infection control should be improved.
\end{abstract}

Keywords: Acinetobacter baumannii; Nosocomial Infection; PDR; XDR

\section{Background}

Acinetobacter baumannii is currently considered to be one of the most important Gram-negative bacteria causing infections in immunecompromised patients, mainly hospitalized in intensive care units (1). A. baumannii is considered to be responsible for $2-10 \%$ of all Gram-negative bacterial infections of patients in intensive care units in Europe and the United States (2).

Infection due to A. baumannii has become a significant challenge for healthcare systems. Several associated mechanisms with carbapenem resistance have been described in A. baumannii, including metallo B-lactamases (MBLs), carbapenem-hydrolysing oxacillinases, decreased permeability due to the loss of outer membrane proteins (OMPs), overexpression of efflux pumps and hyperproduction of AmpC B-lactamases (3). The terms pan drug resistance (PDR), extensive drug resistance (XDR), and multidrug resistance (MDR) are explained, respectively, resistance of a pathogen to all, resistance to all expect 1 or 2 , and resistance to $\geq 3$ classes of antimicrobial agents (4).

\section{Objectives}

The purpose of the present study was to investigate antibiotic susceptibility profile and the rate of XDR or PDR A. baumannii isolated from clinical and environmental specimens of intensive care units of central teaching hospital of Arak University located in center of Iran.

\section{Patients and Methods}

The outbreak occurred in the 350-bed central teaching hospital of Arak University of Medical Sciences in the central part of Iran and a total of 63 non-duplicate A. baumannii isolates were collected from clinical and environ- 
Japoni-Nejad A et al.

mental specimens from March to September 2011. Clinical samples consisted of cerebrospinal fluids ( $n=3,5.4 \%$ ), surgical wound swabs $(\mathrm{n}=4,7.2 \%)$, urine samples $(\mathrm{n}=6$, $10.7 \%$ ), blood samples ( $\mathrm{n}=12,21.4 \%$ ), respiratory secretions ( $n=31,55.3 \%)$ obtained in surgery, neurology and neurosurgery intensive care unit (ICU) wards. Environmental samples of these ICU's were obtained from ventilator tubing (three isolates), suction catheters (two isolates), patient's mattress (one isolate) and the barometer (one isolate). The research was approved by the Ethics Com- mittee of the Faculty of Medicine at the Arak University of Medical Sciences (633).

A. baumannii was identified by Gram staining, colony morphology, motility, cytochrome oxidase reaction, standard biochemical tests and its growth at $43^{\circ} \mathrm{C}$, as well as Microgen kit (Microgen Bioproducts, UK). In addition, phenotypic characterization of $A$. baumannii was confirmed by 16S-rRNA intergenic spacer sequencing (ITS) and OXA-51 PCR (5). The primers and programs can be found in Table 1 .

Table 1. PCR Primers and Cycling Parameters for Genes Presented in this Study

\begin{tabular}{|c|c|c|c|c|}
\hline Gene & Primer/Sequence & PCR Condition & PCR Size & Reference \\
\hline $0 x a 51$ & $\begin{array}{l}\text { F: TAATGCTTTGATCGGCCTTG, R: TGGATTG- } \\
\text { CACTTCATCTTGG }\end{array}$ & $30 \sec 94^{\circ} \mathrm{C}, 40 \sec 52^{\circ} \mathrm{C}, 30 \sec 72^{\circ} \mathrm{C}$ & 353 bp & (6) \\
\hline 16SrRNA & $\begin{array}{l}\text { F: GACGTACTCGCAGAATAAGC, R: TTAGTCTTGCGACCG- } \\
\text { TACTC }\end{array}$ & $1 \min 94^{\circ} \mathrm{C}, 1 \min 58^{\circ} \mathrm{C}, 1 \min 72^{\circ} \mathrm{C}$ & 426 bp & (5) \\
\hline
\end{tabular}

Susceptibilities of the bacterial isolates to a 23 different antimicrobial agents (Mast, UK) were defined by using the standard disk diffusion method according to Clinical and Laboratory Standard Institute (CLSI2011) guidelines and European Committee on Antimicrobial Susceptibility Testing (EUCAST-2011) instructions (Table 2). Susceptibility to Colistin was tested by disk diffusion method using $10 \mu \mathrm{g}$ Colistin sulfate disks. Isolates were considered sensitive if the inhibition zone was $11 \mathrm{~mm}$ or more (7). The results were verified by E-test (bioMérieux, Marcy l'Etoile, France) on Imipenem and Colistin according to CLSI-2011 guidelines. Intermediate sensitivity was considered as resistance.

\begin{tabular}{|c|c|c|}
\hline Antibiotics & Clinical Isolates, No. $(\%)(n=56)$ & Environmental Isolates, No. (\%) $(n=7)$ \\
\hline Aztreonam & $56(100)$ & $7(100)$ \\
\hline Piperacillin & $56(100)$ & $7(100)$ \\
\hline Piperacillin/tazobactam & $56(100)$ & $7(100)$ \\
\hline Amoxicillin/Clavulanic acid & $56(100)$ & $7(100)$ \\
\hline Cefoxitin & $56(100)$ & $7(100)$ \\
\hline Cefotaxime & $56(100)$ & $7(100)$ \\
\hline Ceftazidime & $56(100)$ & $7(100)$ \\
\hline Cefepime & $56(100)$ & $7(100)$ \\
\hline Ciprofloxacin & $56(100)$ & $7(100)$ \\
\hline Levofloxacin & $56(100)$ & $7(100)$ \\
\hline Erythromycin & $56(100)$ & $7(100)$ \\
\hline Clindamaycin & $56(100)$ & $7(100)$ \\
\hline Rifampin & $56(100)$ & $7(100)$ \\
\hline Chlorafenicol & $56(100)$ & $7(100)$ \\
\hline Trimethoprim/Sulfamethoxazole & $56(100)$ & $7(100)$ \\
\hline Netilmicin & $31(56)$ & $3(43)$ \\
\hline Tetracycline & $50(90)$ & $6(86)$ \\
\hline Amikacin & $43(77)$ & $7(100)$ \\
\hline Colistin & $4(7)$ & $3(43)$ \\
\hline Tigecycline $^{\mathrm{a}}$ & $55(98)$ & $7(100)$ \\
\hline Meropenem & $48(86)$ & $5(72)$ \\
\hline Imipenem & $48(86)$ & $5(72)$ \\
\hline Gentamicin & $50(90)$ & $6(86)$ \\
\hline
\end{tabular}

\footnotetext{
$\mathrm{a}$ British (BSAC) tigecycline susceptibility breakpoints (12)
} 
The modified Hodge test (MHT) as recommended by CLSI was used for isolates which showed intermediate or susceptible zones to Imipenem in disk diffusion (8). All isolates were detected for Metallo- $\beta$-lactamases (MBL) production by the E-test MBL strip method; a reduction in the minimum inhibitory concentration

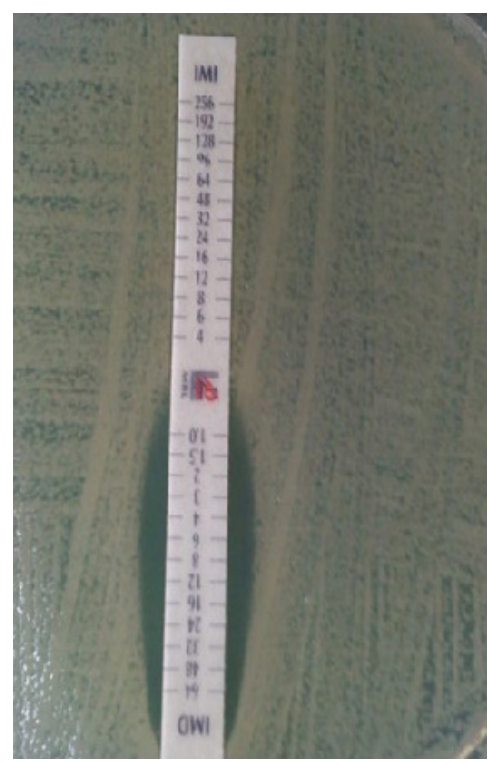

Figure 1. Metallo - $\beta$-lactamase E-test, the Imipenem MIC Decreased From $>256$ to $<1 \mathrm{mg} / \mathrm{L}$ Upon Exposure to a Constant Concentration of EDTA.

\section{Results}

The antimicrobial susceptibility profiles of 63 isolates are shown in Table 2, describing elevated levels of $A$. baumannii resistance, which pose serious treatment challenges. All isolates were resistant to Cefoxitin, Cefotaxime, Ceftazidime, Cefepime, Piperacillin, Piperacillin/ Tazobactam, Amoxicillin/Clavulanic acid, Aztreonam, Ciprofloxacin, Levofloxacin, Erythromycin, Clindamaycin, Chlorafenicol, Rifampin and Trimethoprim/ Sulfamethoxazole. High rates of resistance to Imipenem (84\%), Meropenem (84\%), Gentamicin (89\%), Amikacin (80\%) and Netilmicin (54\%) were observed. Colistin had good antimicrobial activity (89\%).

Of 56 clinical and seven environmental isolates, eight isolates showed intermediate and two isolates revealed susceptible zone to Imipenem, using disk diffusion method. Out of these 8 and 2 isolates, 3 and 1 isolates were positive in the MHT test, respectively. Among all of the $A$. baumannii isolates, 45 (71.4\%) were AmpC $\beta$-lactamase producers and 47 (75\%) were MBL producers analyzed by MBL E-test. The prevalence of PDR and XDR among our isolates was found to be $11 \%$ and $89 \%$. MIC of Colistin and Imipenem are presented in Figure 3.
(MIC) of Imipenem in the presence of ethylene diamine tetra-acetic acid (EDTA) more than or equal to eight-fold was an indicator of MBL activity (Figure 1) (3 , 9). In addition, all isolates were evaluated by AmpC disk test for detection of AmpC betalactamase resistant (Figure 2) $(10,11)$.

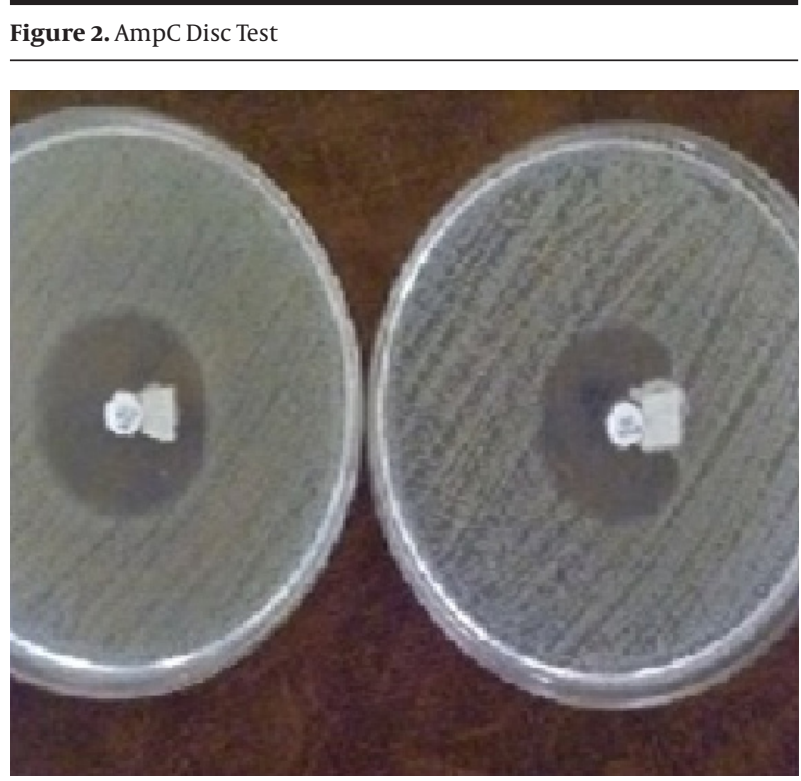

Indentation of the Cefoxitin inhibition zone is observed in the vicinity of the disk with positive strain (Right), while there is an undistorted zone of inhibition near the negative strain (Left).

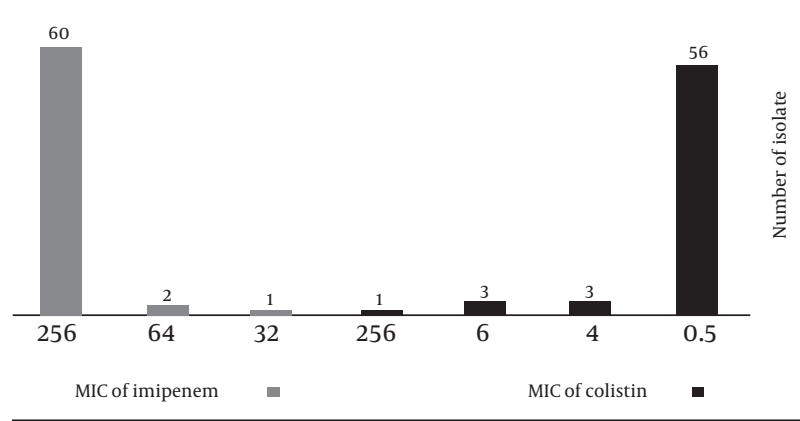

Figure 3. MIC Colistin and Imipenem $(\mathrm{mg} / \mathrm{L})$

\section{Discussion}

Based on these findings, to treat A. baumannii in our hospital, third- and fourth-generation of Cephalosporins are no longer secure. B-lactamase-resistant agents including Piperacillin/Tazobactam and Amoxicillin/Clavulanic acid are not proper choices for treating A. baumannii infections; the Monobactam Aztreonam is an almost ineffec- 
tive antibiotic. Moreover, A. baumannii isolates showed a high level of resistance to Tigecycline and Quinolones (98\% to 100\%) which are due to over-expression of AdeABC efflux pumps and mutation in the Quinolone resistancedetermining region (QRDR) of gyrA and parC genes, respectively (2).

Colistin is only effective against $89 \%$ of $A$. baumannii isolates. Unfortunately, our knowledge on the pharmacokinetics and pharmacodynamics of Colistin are limited, and the current dosage regimens used clinically are based on experience acquired during the past 30 years (13).

In current study, emergence of heterogeneous resistance was confirmed in three strains through E-test and disk diffusion methods for Colistin, isolates presenting colonies inside the inhibition zone were considered to have phenotypic heterogeneous resistance to Colistin (14). Isolated strains are increasingly becoming pan resistant and even those strains that are especially resistant are difficult to treat due to the nephrotoxicity and increasing intermediate sensitivity to Colistin.

In the current study, 53 isolates were identified as Imipenem resistant by disk diffusion method, whereas all clinical and environmental A. baumannii isolates were resistant to Imipenem tested by E-test method, according to CLSI and EUCAST guidelines. Other studies in Asian countries have shown a wide range of A. baumannii Imipenem-resistance in this region including $49 \%$ in Iran, $65 \%$ in Syria and 45\%-90\% in Saudi Arabia (15-17). The presence of six MHT negative isolates, indicating that other mechanism of Carbapenem resistant such as metallobeta-lactamase and AmpC $\beta$-lactamase have also contributed to Carbapenem resistance among these isolates. Out of these six isolates, four were MBL, AmpC producer and two of the other isolates shown to be AmpC positive and have MBL negative pattern.

MBL was produced by 47 (75\%) and AmpC $\beta$-lactamase by 45 (71.4\%) of the Imipenem resistant $A$. baumannii. Therefore, AmpC $\beta$-lactamase and MBL could be an important contributory factor for Imipenem resistance among the isolates in our hospital (11). Modified Hodge test may not be a useful screening test for Carbapenemases as some of MBL producing isolates were not detected by this test (11, 18).

The selective pressure caused by indiscriminate usage of broad-spectrum antibiotics in empirical therapy of hospital infections and environmental contamination are the main reason for such an increased number of patient morbidity and mortality that is related to the infections with the mentioned microorganisms. The MBL, AmpC $\beta$-lactamase and combination of MBL and AmpC production in PDR, XDR A. baumannii isolates are the main reasons of Carbapenem resistance.

\section{Acknowledgements}

This article was extracted from the thesis prepared by
Alireza Japoni-Nejad to fulfill the requirements required for earning the Master of Science degree. We are grateful to Mrs. Marzieh Rahjbaran for support in diagnosis of Acinetobacter isolate.

\section{Authors' Contribution}

For preparing this manuscript, all authors contributed equally.

\section{Financial Disclosure}

Authors declare that there are not any relevant financial interests related to the material in the manuscript.

\section{Funding/Support}

This study was supported by Arak university of Medical Sciences.

\section{References}

1. Ruiz M, Marti S, Fernandez-Cuenca F, Pascual A, Vila J. Prevalence of IS(Aba1) in epidemiologically unrelated Acinetobacter baumannii clinical isolates. FEMS Microbiol Lett. 2007;274(1):63-6.

2. Richet Hervé, Pierre Edouard Fournier MD. Nosocomial infections caused by Acinetobacter baumannii: a major threat worldwide. Infect Cont Hosp Epidemiol. 2006;27(7):645-646.

3. Lin YC, Sheng WH, Chen YC, Chang SC, Hsia KC, Li SY. Differences in carbapenem resistance genes among Acinetobacterbaumannii, Acinetobacter genospecies 3 and Acinetobacter genospecies 13TU in Taiwan. Int J Antimicrob Agents. 2010;35(5):439-43.

4. Falagas ME, Karageorgopoulos DE. Pandrug resistance (PDR), extensive drug resistance (XDR), and multidrug resistance (MDR) among Gram-negative bacilli: need for international harmonization in terminology. Clin Infect Dis. 2008;46(7):1121-2.

5. Lin L, Ling BD, Li XZ. Distribution of the multidrug efflux pump genes, adeABC, adeDE and adeIJK, and class 1 integron genes in multiple-antimicrobial-resistant clinical isolates of Acinetobacter baumannii-Acinetobacter calcoaceticus complex. Int $J$ Antimicrob Agents. 2009;33(1):27-32.

6. Woodford N, Ellington MJ, Coelho JM, Turton JF, Ward ME, Brown S, et al. Multiplex PCR for genes encoding prevalent OXA carbapenemases in Acinetobacter spp. Int J Antimicrob Agents. 2006;27(4):351-3.

7. Gales AC, Reis AO, Jones RN. Contemporary assessment of antimicrobial susceptibility testing methods for polymyxin B and colistin: review of available interpretative criteria and quality control guidelines. J Clin Microbiol. 2001;39(1):183-90.

8. Clinical Laboratory Standards Institute. Performance standards for antimicrobial susceptibility testing, twenty first informational supplement M100-S21. 2011;CLSI Wayne, PA

9. Segal H, Elisha BG. Use of Etest MBL strips for the detection of carbapenemases in Acinetobacter baumannii. J Antimicrob Chemother. 2005;56(3):598.

10. Hanson ND. AmpC beta-lactamases: what do we need to know for the future? J Antimicrob Chemother. 2003;52(1):2-4.

11. Noyal MJ, Menezes GA, Harish BN, Sujatha S, Parija SC. Simple screening tests for detection of carbapenemases in clinical isolates of nonfermentative Gram-negative bacteria. Indian J Med Res. 2009;129(6):707-12.

12. Brink AJ, Bizos D, Boffard KD, Feldman C, Grolman DC, Pretorius J, et al. Guideline: appropriate use of tigecycline. S Afr Med J. 2010;100(6 Pt 2):388-94.

13. Li J, Rayner CR, Nation RL, Owen RJ, Spelman D, Tan KE, et al. Heteroresistance to colistin in multidrug-resistant Acinetobacter baumannii. Antimicrob Agents Chemother. 2006;50(9):2946-50.

14. Fernandez Cuenca F, Sanchez Mdel C, Caballero-Moyano FJ, Vila J, Martinez-Martinez L, Bou G, et al. Prevalence and analysis of microbiological factors associated with phenotypic heterogeneous 


\section{Japoni-Nejad A et al.}

resistance to carbapenems in Acinetobacter baumannii. Int J Antimicrob Agents. 2012;39(6):472-7.

15. Al Johani SM, Akhter J, Balkhy H, El-Saed A, Younan M, Memish Z. Prevalence of antimicrobial resistance among gramnegative isolates in an adult intensive care unit at a tertiary care center in Saudi Arabia. Ann Saudi Med. 2010;30(5):364-9.

16. Feizabadi MM, Fathollahzadeh B, Taherikalani M, Rasoolinejad M, Sadeghifard N, Aligholi M, et al. Antimicrobial susceptibility patterns and distribution of blaOXA genes among
Acinetobacter spp. Isolated from patients at Tehran hospitals. Jpn J Infect Dis. 2008;61(4):274-8.

17. Hamzeh AR, Al Najjar M, Mahfoud M. Prevalence of antibiotic resistance among Acinetobacter baumannii isolates from Aleppo, Syria. Am J Infect Control. 2012;40(8):776-7.

18. Maragakis LL, Perl TM. Acinetobacter baumannii: epidemiology, antimicrobial resistance, and treatment options. Clin Infect Dis. 2008;46(8):1254-63. 